\title{
NEW EVOLUTIONARY TRACKS OF PRE-MAIN-SEQUENCE STARS
}

\author{
Francesco Palla and Steven W. Stahler \\ Osservatorio di Arcetri, Largo Fermi 5, 50125 Firenze, Italy \\ Giacomo Parigi \\ Dipartimento di Astronomia, Università di Firenze, Largo Fermi 5, 50125 \\ Firenze, Italy
}

\begin{abstract}
We present the results of new evolutionary calculations of the pre-main-sequence phase of stars in the mass range 1 to $5 \mathrm{M}_{\odot}$. The novelty of our approach is that, unlike in the standard theory of PMS evolution, the initial conditions of the gravitational contraction phase are not arbitrarily set, but derive from protostellar theory.
\end{abstract}

\section{INTRODUCTION}

The theory of star formation provides the appropriate conditions for stars at the beginning of the PMS phase. However, although this statement has been frequently advocated in the literature (e.g. Bisnovatyi-Kogan 1981), standard calculations have so far ignored this critical point and assumed ad hoc initial conditions. Since the classical works of Hayashi, Hoshi \& Sugimoto (1962), Iben (1965), and Ezer \& Cameron (1965), it has been a common procedure to assume that stars of all masses begin the PMS phase as fully convective objects, high up on the Hayashi track. Based on simple energy conservation arguments, Cameron (1962) estimated that $R_{*}^{\text {init }} \sim 50 \mathrm{R}_{\odot}\left(\mathrm{M}_{*} / \mathrm{M}_{\odot}\right)$. Thus, convection is due to, and maintained by, the large stellar radius and low effective temperatures (Bodenheimer 1966, cf. also Staller 1988 for a general discussion).

The recent progress in the understanding of protostellar evolution justifies a critical evaluation of some of the basic assumptions of PMS theory. In fact, detailed calculations of the protostellar accretion phase have shown that not only the initial radius, but also the internal structure of stars present significant differencies from the standard models. In the following, we present new evolutionary tracks computed using the results of protostellar models. Let us start with a brief summary of the main features of protostellar evolution.

\section{PROTOSTELLAR EVOLUTION}

The key properties of protostars are determined by the action of deuterium burning. For low-mass stars $\left(\mathrm{M}_{*} \leq \mathrm{M}_{\odot}\right)$, nuclear burning takes place in the center and keeps the star fully convective (Stahler 1988). For more massive stars, deuterium is burned in a subsurface shell, that can comprise a substantial fraction of the whole mass, while the interior is in a state of radiative equilibrium 
(Palla \& Stahler 1990). Independently of the details of the accretion flow, the protostellar evolution from low- to intermediate-mass stars is found to proceed through the following series of events: $D$-burning in the center; appearance of a radiative barrier; exhaustion of central D; ignition of D-burning in a shell; rapid swelling of the star; final contraction to the main-sequence (Palla \& Stahler 1992a). These processes determine the variation of the protostellar radius with mass. The mass-radius relation is the fundamental input provided by star formation theory to PMS evolution. The key result is that during the accretion phase, protostars never achieve radii as large as those predicted by Cameron's estimate (Hayashi-type initial conditions), but typically remain a factor of 10 smaller. Also, convection is not due to surface conditions, but rather depends entirely on the nuclear activity of the star. In the assumption that accretion ends rapidly, the mass-radius relation gives a theoretical birthline, i.e. the locus in the $\mathrm{H}-\mathrm{R}$ diagram where stars of different mass should appear when they become optically visible as PMS stars. Thus, the initial conditions are determined self-consistently.

\section{PRE-MAIN-SEQUENCE EVOLUTION}

We have followed the PMS evolution of stars of $\mathrm{M}_{*}=1,2,3.5,4,5 \mathrm{M}_{\odot}$. For each mass, the initial values of the radius and effective temperature are those given at the birthline. In all calculations, the chemical composition is $\mathrm{X}=0.70$, $Z=0.02$, and the $[D / H]$ ratio is taken to be the interstellar value $2.5 \times 10^{-5}$ by number. Convection is treated using mixing length theory, with a value of the ratio $l / H_{p}=1.5$. Full details of the input physics can be found in Parigi (1992) and Palla \& Stahler (1992b, in preparation). The computed set of tracks in the $H-R$ diagram is shown in Figure 1 . The main results can be summarized as follows:

$M_{*}=1 M_{\odot}$, the star begins the evolution while actively burning deuterium in the center, and moves up along the Hayashi track. The main D-burning phase is substantially reduced with respect to previous calculations. The effects of nuclear burning on the pulsational instability of the star have been analyzed by Parigi (1992). The amplification factors of the pulsation have been found to be 4 to 7 times smaller than that computed by Toma (1972); thus, it is unlikely that oscillations have time to develop before D-burning ceases.

$1 \leq M_{*} / M_{\odot} \leq 2$, the Hayashi phase is quite short. Convection is due to $D$-burning in the center, but the fraction of $D$ available is very small and the duration of the nuclear phase is correspondingly short.

$2 \lesssim M_{*} / M_{\odot} \leqslant 4$, stars do not show a Hayashi phase at all. The initial evolution is opposite to that predicted by standard models: stars begin the PMS phase from low luminosities and join the radiative tracks from below.

$\mathrm{M}_{*} \geq 4 \mathrm{M}_{\odot}$, stars begin the evolution already on the radiative portion of the evolutionary track, close to the Main Sequence. The evolutionary lifetimes can be reduced by as much as $40 \%$ with respect to previous estimates (cf. Table 1). We do not expect stars more massive than $8 \mathrm{M}_{\odot}$ to have a PMS phase at all, i.e. such stars are born directly on the ZAMS. 


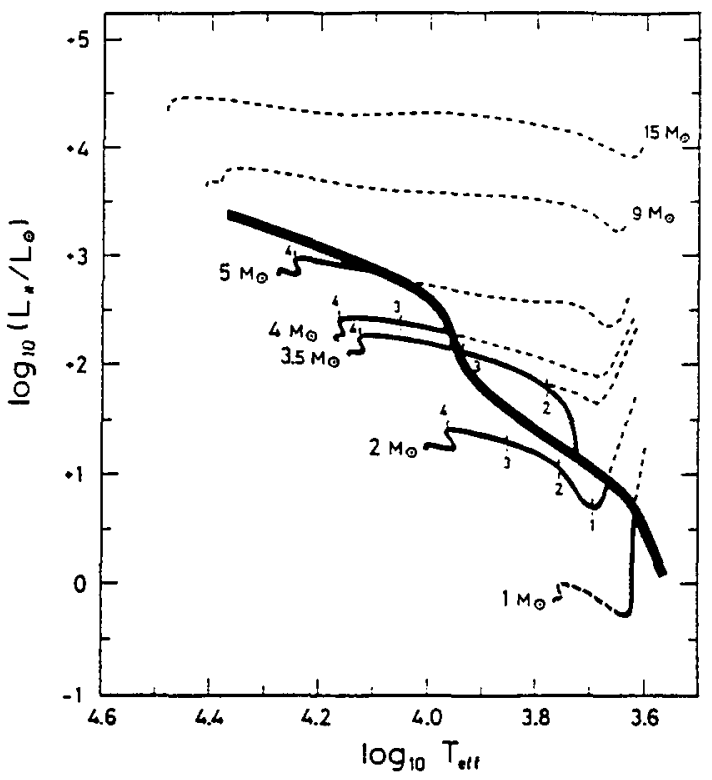

Fig. 1. PMS evolutionary tracks for stars of mass $1 \mathrm{M}_{\odot} \leq \mathrm{M}_{*} \leq 5 \mathrm{M}_{\odot}$ (solid lines). Evolutionary lifetimes are marked on each curve. The heavy solid line represents the "birthline". For comparison, the dashed lines give the standard tracks as computed by Iben (1965).

Evolutionary lifetimes (in years)

\begin{tabular}{ccccc}
\hline \hline Point & $1 M_{\odot}$ & $2 M_{\odot}$ & $3.5 M_{\odot}$ & $4 M_{\odot}$ \\
\hline 1 & $1.0 \times 10^{7}$ & $1.6 \times 10^{6}$ & - & - \\
2 & - & $3.6 \times 10^{6}$ & $2.8 \times 10^{5}$ & - \\
3 & - & $4.6 \times 10^{6}$ & $5.3 \times 10^{5}$ & $4.3 \times 10^{5}$ \\
4 & - & $5.4 \times 10^{6}$ & $7.1 \times 10^{5}$ & $5.6 \times 10^{5}$ \\
\hline
\end{tabular}

\section{REFERENCES}

Bisnovatyi-Kogan, G.S. 1981, in Fundamentals Problems in the Theory of Stellar Evolution, IAU Symp. N. 93, eds. D. Sugimoto et al., D. Reidel Publ. Co., 85.

Bodenheimer, P. 1966, Ap. J., 144, 709.

Cameron, A.G.W. 1962, Icarus, 1, 13.

Ezer, D. \& Cameron, A.G.W. 1965, Can. J. Phys., 43, 1497.

Hayashi, C., Hoshi, R. \& Sugimoto, D. 1962, Progr. Theor. Phys. Suppl. 22.

Iben, I. 1965, Ap.J., 141, 993.

Palla, F. \& Stahler, S.W. 1990, Ap. J., 360, L47.

Palla, F. \& Stahler, S.W. 1992a, Ap. J., in press.

Parigi, G. 1992, Theisis, Univerisy of Florence.

Stahler, S.W. 1988, P.A.S.P., 100, 1474.

Toma, E. 1972, A\&A, 19, 76. 